\title{
Modelling the influences of climate change-associated sea-level rise and socioeconomic development on future storm surge mortality
}

Simon J Lloyd

R Sari Kovats

Zaid Chalabi

Department of Social and Environmental Health Research, London School of Hygiene and Tropical Medicine, 15-17 Tavistock Place, London. WC1H 9SH. United Kingdom.

Sally Brown

Robert J Nicholls

Faculty of Engineering and the Environment and Tyndall Centre for Climate Change Research, University of Southampton, University Road, Highfield, Southampton. SO17 1BJ., United Kingdom

Corresponding author: Sari Kovats

Phone: +44 (0) 2079272962

sari.kovats@1sthm.ac.uk

Article Note: This project was funded by the Natural Environment Research Council under the QUEST (Quantifying and Understanding the Earth System) project: contract numbers $\mathrm{NE} / \mathrm{E} 001874 / 1$ and NE/E001882/1. For the sea level rise projections, we thank the international modelling groups for providing their data for analysis, the Program for Climate Model Diagnosis and Intercomparison (PCMDI) for collecting and archiving the model data, the JSC/CLIVAR Working Group on Coupled Modelling (WGCM) and their Coupled Model Intercomparison Project (CMIP) and Climate Simulation Panel for organising the model data analysis activity, and the IPCC WG1 TSU for technical support. The IPCC Data Archive at Lawrence Livermore National Laboratory is supported by the Office of Science, US Department of Energy. For the flood disaster data, we thank EM-DAT: The OFDA/CRED International Disaster Database at the Université Catholique de Louvain, Brussels, Belgium (www.emdat.be). 
Abstract: Climate change is expected to affect health through changes in exposure to weather disasters. Vulnerability to coastal flooding has decreased in recent decades but remains disproportionately high in lowincome countries. We developed a new statistical model for estimating future storm surge-attributable mortality. The model accounts for sea-level rise and socioeconomic change, and allows for an initial increase in risk as low-income countries develop. We used observed disaster mortality data to fit the model, splitting the dataset to allow the use of a longer time-series of high intensity, high mortality but infrequent events. The model could not be validated due to a lack of data. However, model fit suggests it may make reasonable estimates of log mortality risk but that mortality estimates are unreliable. We made future projections with and without climate change (A1B) and sea-based adaptation, but given the lack of model validation we interpret the results qualitatively. In low-income countries, risk initially increases with development up to mid-century before decreasing. If implemented, sea-based adaptation reduces climate-associated mortality in some regions, but in others mortality remains high. These patterns reinforce the importance of implementing disaster risk reduction strategies now. Further, while average mortality changes discontinuously over time, vulnerability and risk are evolving conditions of everyday life shaped by socioeconomic processes. Given this, and the apparent importance of socioeconomic factors that condition risk in our projections, we suggest future models should focus on estimating risk rather than mortality. This would strengthen the knowledge base for averting future storm surge-attributable health impacts.

Keywords: coastal floods, climate change, mortality, sea-level rise, quantitative modelling Abbreviations: DIVA: Dynamic Interactive Vulnerability Assessment; EM-DAT: Emergency Events Database; GCM: General Circulation Model; GDP: Gross Domestic Product; HDI: Human Development Index; LHS: Left-Hand Side; RHS: RightHand Side; SLR: Sea-Level Rise; UN: United Nations; UNDP: United Nations Development Programme WPP: World Population Prospects

\section{Introduction}

Climate change is expected to affect health through changes in exposure to weather disasters, including via wind storms (e.g. cyclones) ${ }^{1}$ and coastal flooding (IPCC, 2012). During 19802000, cyclones caused an average of 12,000 deaths per year globally (Shultz et al., 2005), but a single disaster can cause a large loss of life in the absence of adequate defences and/or warning systems. High mortality events often occur in lower-income countries, but highincome countries are not immune: Cyclone Nargis caused 138,000 deaths in Burma in 2008

\footnotetext{
${ }^{1}$ Throughout, we use "cyclones" as a general term to refer to any major wind storm that may be associated with a storm surge; e.g. extra-tropical storms, hurricanes, tropical cyclones, typhoons etc.
} 
(Fritz et al., 2009), and Hurricane Katrina caused 1,800 deaths in the USA in 2005 (Knabb et al., 2006).

Vulnerability to cyclones has decreased in recent decades due to improved disaster preparedness, but vulnerability remains about 200 times greater in low-income than in higher-income countries (UNISDR, 2011). Further, risk does not decline linearly with economic development: observations suggest that as low income countries develop, risk may initially increase before decreasing (De Haen and Hemrich, 2007, Kellenberg and Mobarak, 2008). For example, expansion of slums in coastal cities may increase population exposure at a greater pace than can be compensated by protective measures.

Cyclone mortality is associated with high-speed winds, heavy rains, and storm surge. We focus on mortality risk associated with storm surge, defined as sea water pushed forward and drawn up by a depression which floods an otherwise dry area of land. Climate change is expected to worsen storm surge events through sea-level rise (Brown et al., 2013) and via an increase in intensity, but not frequency, of cyclones (IPCC, 2012, Emanuel, 2005). Nonclimate factors will also affect future surge risk including physical changes such as land subsidence, and socioeconomic changes such as increased coastal population (McGranahan et al., 2007) and disaster preparedness.

Previous studies of future flood mortality are of two types: 'event-based' (see Jonkman et al., 2008 for a review; also, Penning-Roswell et al., 2005, Maaskant et al., 2009) or 'average mortality' (e.g. McMichael et al., 2004, Peduzzi et al., 2012) models. The former focus on single flood events, using detailed data describing flood characteristics (e.g. depth, velocity), area-specific conditions (e.g. buildings, evacuation routes), and the exposed population (e.g. age distribution). The data requirements mean the strategy is not suitable for global-level modelling. 'Average-mortality' models consider a given area (e.g. a grid cell, a nation-state) and use data on long term probabilities of events, average population exposure, and average socioeconomic conditions to estimate average mortality. We adopt this latter strategy.

To our knowledge, only two papers have quantified global storm-surge mortality. McMichael et al (2004) developed a model using a 20 year series of mortality data for all coastal flood disasters. Mortality risk was estimated using national population as the denominator. For 
future projections, the changes in population vulnerability were linearly scaled to Gross Domestic Product (GDP) per capita.

Dasgupta et al (2009) developed a spatially explicit mortality model for 84 countries and 577 coastal cities. They modelled 1:100-year storm surges, and assessed future impacts under climate change accounting for sea-level rise and a $10 \%$ increase in event intensity. Despite detailed physical modelling, socioeconomic changes were poorly represented: future countrylevel impacts assumed no population or socioeconomic changes from the present, and citylevel impacts held socioeconomics constant but accounted for population change.

In this paper, we developed a new statistically-based 'average mortality' model for estimating future mortality attributable to storm surge due to climate change in the context of socioeconomic change.

First, we describe the coastal flood model (DIVA) which provides the principal input into our mortality risk model: population at risk of exposure to storm surge. Second, we outline the development of the mortality risk model. Third, we describe model calibration. Fourth, we project future mortality risk and mortality under given climate and socioeconomic scenarios.

\section{Coastal flood model}

The Dynamic Interactive Vulnerability Assessment (DIVA) is an integrated bio-geophysical model (Vafeidis et al., 2008, Hinkel and Klein, 2009) which assesses the impacts of sea-level rise (assuming no increase in storminess), subsidence and socio-economic change in the coastal zone. Pattern-scaled climate scenarios were derived from Brown et al (2013) (also see Section 5.1.1), subsidence from Peltier (2000b, Peltier, 2000a), and socioeconomics (population and GDP) from the SRES socio-economic scenarios (Nakicenovic and Swart 2000) (see Online Resource, ESM, Appendix S1).

We used the DIVA country-level output, "average annual people at risk of exposure to storm surge"; i.e. expected number of people flooded per year if they do not evacuate or move to storm shelters, as analysed in Brown et al (2013). 
DIVA considers two engineered adaptation strategies ('sea-based strategies'). 'No upgrade to protection' models dikes for a common baseline (1995) and assumes this standard of protection is not upgraded as sea-level rises and socioeconomics change; i.e. a future without adaptation. 'Upgrade to protection' entails that dikes are upgraded reflecting changes in population density as sea-level rises, and that there is beach nourishment in response to erosion; i.e. a future with adaptation.

Two aspects of DIVA guided the development of the mortality risk model. Firstly, DIVA considers sea-based strategies of adaptation and estimates people at risk of flooding if the defences are breached. It does not account for other adaptation strategies such as warning systems, shelters, and building regulation ('land-based strategies'). We therefore included an analogue of the Human Development Index (HDI) (UNDP, 2010) as a proxy variable for land-based strategies in the mortality risk model. That is, we refer to two types of adaptation: 'sea-based strategies' (as modelled by DIVA), and 'land-based strategies' (as modelled in the mortality risk model).

Secondly, DIVA assumes that cyclone intensity and frequency will remain at baseline levels in the future. Thus, the mortality risk model assumes the same.

\section{Mortality risk model}

\subsection{Form of the model}

Population mortality risk is a function of climatic and socioeconomic conditions. To model this, we adopted a model structure based on Patt et al (2010), who developed a statistical model for estimating country-level vulnerability (as log mortality risk) to climate-related extreme events. We tested various configurations of the model and selected the following form (see Section 4):

$$
\ln \left[\frac{M_{i, j}+1}{X_{i, j}} \times 10^{6}\right]=\beta_{1} \times \ln \left(E_{i}+1\right)+\beta_{2} \times \ln \left(P_{i, j}\right)+\beta_{3} \times H_{i, j}+\beta_{4} \times H_{i, j}^{2}+k
$$

where:

$M_{i, j}$ is average annual surge mortality in country $i$, in time-slice $j$, 
where $j$ is 2000 for calibration, and 2030, 2050, and 2080 for future projections $X_{i, j}$ is average annual people at risk of exposure to storm surge (as estimated by DIVA) ' $10^{6}$ ' scales the equation to 'per million people exposed' $E_{i}$ is average annual number of surge events $P_{i, j}$ is national population $H_{i, j}$ is an analogue of the HDI $\beta_{a}$ are fitted parameters, where $a=1$ to 4 $k$ is the fitted constant

Following DIVA, which holds event frequency and intensity constant, we hold $E_{i}$ constant over time. $M_{i, j}$ and $E_{i}$ were shifted by 1 as they may take zero values meaning the log term would be undefined.

The future time-slices are 2026 to 2030,2046 to 2050 , and 2076 to 2080 for $j=2030,2050$ and 2080 respectively. The baseline time-slice $(j=2000)$ used for calibration represents the present. Typically when conducting a multi-model assessment, data from various sources are not strictly aligned: exposure data are for 1996 to 2000; mortality data cover 1970 to 2010; and socioeconomic data are for 2000.

The left-hand side (LHS) of equation (1) approximates to 'log mortality risk per million people at risk of exposure to storm surge' when mortality is high (as shifting mortality by 1 would have little influence). Hence we refer to the LHS as 'log mortality risk'.

Following Patt et al (2010), the variables on the right-hand side (RHS) are interpreted as follows. $E_{i}$ and $P_{i, j}$ represent exposure characteristics. As the number of annual events $\left(E_{i}\right)$ increases, coping capacity is expected to decrease, and hence average mortality risk is expected to increase. Conversely, it is 'expected that larger countries are likely to experience disasters over a smaller proportion of their territory or population, and also benefit from potential economies of scale in their disaster management infrastructure' (Patt et al., 2010); thus as population $\left(P_{i, j}\right)$ increases, mortality risk is expected to decrease. 
The HDI $\left(H_{i, j}\right)$ is a national-level measure of development accounting for social and economic factors (UNDP, 2010). It takes values from 0 to 1 , where 0 is the lowest level of development and 1 the highest. Here, the HDI acts a proxy for land-based strategies of adaptation.

Generally, as $H_{i, j}$ increases, mortality risk may be expected to decline. However, for coastal floods, observations suggest that as low income countries develop, risk initially increases (see Section 1). Because of this, the model includes $H_{i, j}$ as a quadratic term. Due to data availability, we adopted an analogue of the HDI (see Section 4.1.3).

\subsection{Extraction of future mortality estimates}

Equation (1) estimates future mortality risk. Estimates of future mortality $\left(M_{i, j}\right)$ are extracted by rearranging equation (1):

$$
M_{i, j}=\left[\frac{\exp (R H S) \times X_{i, j}}{10^{6}}\right]-1
$$

where:

$$
R H S=\beta_{1} \times \ln \left(E_{i}+1\right)+\beta_{2} \times \ln \left(P_{i, j}\right)+\beta_{3} \times H_{i, j}+\beta_{4} \times H_{i, j}^{2}+k
$$

Note that as the final step is to subtract 1 , it is possible to obtain country-level results where $-1 \leq M_{i, j}<0$. In these instances results are rounded up to 0 . Additionally, if predicted mortality exceeded exposed, we set mortality equal to exposure. In our projections, this was necessary for three islands in Oceania. It is likely this problem arose due a combination of model error and locations with low exposure (due to low populations) but high risk. 


\section{Model calibration}

We drew on various data sources and performed a number of transformations to calibrate the model (See Figure 1). In some instances, the same data enters the model in multiple locations; this situation is not uncommon in integrated assessment methods and we discuss the implications in Online Resource, ESM, Appendix S2.

\subsection{Data for fitting the model}

\subsubsection{Baseline storm surge exposure $\left(X_{i, 2000}\right)$}

Observational data for surge exposure are not available. We used modelled national-level estimates of exposure from DIVA for average annual exposure for 1996 to 2000. Globally, about 3.5 million people were at risk of exposure annually (see Online Resource, ESM, table $\mathrm{S} 1$ for regions, and table S2 for regional baseline exposure). As these were the only available exposure estimates we fit equation (1) cross-sectionally for a single time-slice.

\subsubsection{Baseline storm surge-attributable mortality $\left(M_{i, 2000}\right)$}

Data for baseline storm surge-attributable mortality are not available. Thus we derived estimates from the only accessible global disaster dataset: the Emergency Events Database (EM-DAT) (CRED, 2011).

EM-DAT provides mortality data by event by country. An event is included if: 10 or more people are killed; 100 or more are people affected; a state of emergency is declared; or, a call is made for international assistance. EM-DAT reports total mortality for cyclone events, and storm surge-specific deaths are not available. Consequently, we extracted all cyclone events (classified as Hydrological: storm surge/coastal flood; Meteorological: extratropical/tropical cyclone) over the period 1970 to 2010. For unclassified events, we checked other fields in the database (e.g. named events are likely to be cyclones). We identified a total of 1,569 events. 
A large proportion of total cyclone mortality is attributable to large infrequent events. Therefore it is preferable to assess average mortality using a long time-series of mortality data. EM-DAT data quality has improved in recent years, and may be considered reasonably complete for 15 to 20 years. However, events with high mortality may have been reasonably well recorded for around 30 to 40 years (personal communication, Phillipe Hoyois, EMDAT).

This presents a trade-off. On one hand, restricting data to the last 20 years would maximise completeness, but may introduce considerable biases: if an infrequent but high impact event struck a country during this period, average annual deaths would be high; if it was not struck, average deaths would be too low. On the other hand, using data covering 40 years would provide better (although not optimal) coverage of high impact events, but would exclude many smaller events.

The best use of the data was to create two data sets: a short time-series covering 1990 to 2010 including only 'small' events $(s=1 \ldots S)$, and a long time-series covering 1970 to 2010 including only 'large' events $(l=1 \ldots L)$. After inspecting the data, we defined 'small' events as those with less than 200 deaths, and 'large' events as those with 200 or more deaths.

As exposure data for the present were available only as averages for the baseline time-slice, we required corresponding mortality estimates. Our mortality data covered the period from 1970 onward; during this time, world population almost doubled (United Nations, 2011) and this would have influenced death tolls. Thus to bring exposure and mortality data into line, we standardized deaths in all events to population in the year 2000 using standard methods (e.g. Donaldson and Donaldson, 2003). (See Online Resource, ESM, Appendix S3 for details and regional-level event and mortality data).

We then estimated the fraction of all-cause cyclone-attributable deaths that were attributable to storm surge. We assumed that in the least developed countries about $90 \%$ of cyclone mortality is surge-attributable (Rappaport, 2000) compared to about 67\% in more developed countries (Jonkman, 2005, Jonkman et al., 2009). We estimated baseline storm surgeattributable mortality $\left(M_{i, 2000}\right)$ using a piecewise linear function and the HDI-analogue $\left(H_{i, 2000}\right)$ (See Online Resource, ESM, Appendix S4). 
Finally, we combined mortality and exposure to estimate average annual mortality risk for the baseline time-slice. (See Online Resource, ESM, Table S3, for baseline surge-specific mortality and mortality risk estimates).

\subsubsection{Mortality risk model input variables $\left(E_{i}, P_{i, 2000}, \mathrm{H}_{\mathrm{i}, 2000}\right)$}

We estimated $E_{i}$, the average annual number of cyclone events in country $i$, by summing the average annual number of events in the 'small' and 'large' event datasets (See Online Resource, ESM, Appendix S3, table S3.1). Populations in the year 2000, $P_{i, 2000}$, were from the World Population Prospects (WPP), 2010 Revision (United Nations, 2011). Following Patt et al (2010), when calibrating the model (see Section 4.2) we tested a variable for fertility (also from the WPP), used as an indicator of women's empowerment.

The HDI is the geometric mean of normalised estimates of GDP/capita, life expectancy at birth, and education (UNDP, 2010). For consistency between baseline data and future projections we used an analogue of the HDI. GDP data was from the World Bank Development Indicators (World Bank, 2012), population and life expectancy data from the WPP (United Nations, 2011), and 'years of education at the age of 25' from Barro and Lee (2000). All data was for the year 2000, or the nearest year available (See Online Resource, ESM, Appendix S5 for the method for calculating the HDI-analogue).

\subsection{Calibration}

We calibrated the mortality risk model using data for 141 countries with baseline data. After testing various forms we adopted equation (1), which met the following criteria. Firstly, the parameterized equation had a good statistical fit (adjusted $R^{2}=0.43$ ) (Table 1). Secondly, in conceptual terms (see Section 3.1), the signs of the estimated parameters are as expected. $\beta_{1}$ is positive, meaning risk increases as events increase; $\beta_{2}$ is negative, meaning risk decreases as population increases; and $\beta_{4}$ is negative, meaning the equation is concave in relation to the HDI-analogue. Finally, it appeared to be fit-for-purpose (i.e. for making future projections) as the standardized regression coefficients show the equation is most responsive 
to variables for which the most reliable projection data are available. That is, the model is most sensitive to changes in 'development' $\left(H_{i, j}\right)$ and population $\left(P_{i}\right)$ and least responsive to 'events' $\left(E_{i}\right)$, which is in approximation and held constant over time. (For further details, see Online Resource, ESM, Appendix S6).

Classically, we would validate the calibrated model using an independent dataset. We were unable to do so for two reasons. Firstly, as only 141 national 'observations' were available we used all these data to calibrate the model. Secondly, prior to calibrating the model, the majority of the data were transformed (see Figure 1) meaning that even if a sub-set of data were reserved for validation it would not have been independent. Hence, the best available indicator of model fit was the adjusted $R^{2}$. While this suggests log mortality risk is predicted reasonably well, it does not indicate the model makes reliable future projections.

For mortality, we compared 'observed' mortality $\left(M_{i, 2000}\right)$ with estimates extracted using equation (2). Correlation was poor ( $R=0.08$ ), suggesting mortality estimates are not reliable. This is partially because the model was fit in the logarithmic space (see Online Resource, ESM, Appendix S7). Total global 'observed' average annual mortality is 23,900 compared to 14,600 predicted by the model.

\section{Future projections}

We estimated future storm surge-attributable mortality risk and mortality with land-based adaptation, with and without climate change-associated sea-level rise, and with and without sea-based strategies of adaptation, for the 2030s, 2050s and 2080s.

\subsection{Scenarios and future exposure estimates}

\subsubsection{Scenarios}

The 'with climate change' scenario was modelled for an A1B future using seven General Circulation Models (GCMs) (Online Resource, ESM, Table S3) with an assumption of no change in storminess (Brown et al., 2013). Across the GCMs, global mean sea-level rise 
ranged from $0.28 \mathrm{~m}$ to $0.53 \mathrm{~m}$ by 2100 , with respect to $1961-1990$. A sea-level rise scenario for a 'without climate change' scenario was also derived.

GDP and population data for an A1B scenario were from IMAGE 2.3 (van Vuuren et al., 2007). Rates of population growth in the coastal zone were assumed to be the same as the national growth rates. For the HDI-analogue, life expectancy data were from the WPP (United Nations, 2011), and years of education at age 25 were from Barro and Lee (2000). (See Online Resource, ESM, Table S4).

\subsubsection{Future exposure estimates $\left(\mathrm{X}_{\mathrm{i}, \mathrm{j}}\right)$}

DIVA estimated future national-level average annual people at risk of exposure to storm surge in futures with and without climate change, and with and without sea-based strategies of adaptation (All future projections include land-based adaptation). In futures with climate change, the median estimate across the GCMs was used as the exposure estimate $\left(X_{i, j}\right)$.

\subsection{Results}

We estimated future log mortality risk at the national-level, and mortality for 21 regions (see Online Resource, ESM, table S1). Given the model was not validated, and that it is known that mortality predictions are poor, the results should be seen as indicative at best. Of more interest are: (i) how future mortality risk changes with different input variables, and, (ii) how mortality patterns may change in futures with and without climate change, and with and without sea-based adaptation.

In the projections it was possible for surge exposure to be 0 ; here, to avoid division by 0 , we set the LHS of equation (1) to 0. (i.e. if exposure to surge is zero, mortality risk must also be $0)$.

\subsubsection{Log mortality risk}

We estimated future log mortality risk at the national level (We did not aggregate this to regional level as it is not additive; $\log (a)+\log (b)=\log (a \times b))$. Quantitative estimates of $\log$ 
mortality risk are difficult to interpret, but qualitative patterns show how factors included in the model interact to shape changes in mortality risk over time.

Figure 2 shows projected log mortality risk estimates made using equation 1 for four selected countries chosen to illustrate diverse patterns. In each plot, the colour contours represent log mortality risk (i.e. LHS of equation 1) for that country, as a function of factors on the RHS of equation 1: the HDI-analogue ( $H_{i, j}, \mathrm{x}$-axis) and national population ( $P_{i, j}, \mathrm{y}$-axis). Note that the pattern of contours differs for each country due to the influence of number of annual events $\left(E_{i}\right)$. The black dots indicate the projected trajectory of log mortality risk over the next century, assuming climate change and land-based adaptation but without sea-based adaptation. Each dot is labelled with the time-slice (in brackets) and projections of average annual surge exposure $\left(X_{i, j}\right)$, which is a function of sea-level rise, land subsidence and population living in the coastal zone.

In Bangladesh, despite an increasing HDI, risk increases until 2050 but then decreases to 2030 levels in 2080 as HDI continues to increase. That is, the benefits of improved landbased strategies in the 2080s are partially off-set by increased exposure risk. In Mozambique there is an even larger increase in mortality risk out to 2050 as HDI rises from a very low to moderate level. After this risk may rise further before returning to 2050 levels in 2080; again, rises in exposure partially off-set benefits associated with land-based adaptation and increases in population.

In the USA, risk continually decreases, largely because population increases. This is despite more than a 10-fold increase in exposure by 2080; that is, population increases off-set exposure increases. Finally, Jamaica illustrates the difficulties of estimating baseline exposure risk in high-risk small islands; the estimated average exposure of 10 per year is likely to be too low. However, the model suggests baseline risk amongst the exposed is high, and that despite increases in exposure with time, risk declines - although it remains high - as the HDI increases. 


\subsubsection{Mortality}

As the mortality projections are not robust we provide estimates of future mortality at regional level as categorical estimates. Figure 3 shows regional mortality at baseline and in the 2030, 2050 and 2080; regions are shown on the vertical axis, and time-slice on the horizontal axis. For each region there are three bars. The upper bar shows mortality without climate change and without sea-based strategies of adaptation. The central bar shows a future with climate change but without (sea-based) adaptation; comparing it with the top bar gives an indication of climate change-attributable mortality. The lower bar shows mortality in futures with climate change and adaptation; comparing the middle and lower bars gives an indication of the mortality burden avoidable via adaptation. (All futures include land-based strategies of adaptation).

The results suggest that in East Asia, mortality will increase over time without climate change (upper bar), but that climate change will increase mortality further by 2080 (central bar). Sea-based strategies of adaptation may reduce future mortality to around baseline levels (lower bar). Similar patterns are seen in the Caribbean, Oceania, and Eastern and Western Sub-Saharan Africa. In these regions, adaptation reduces mortality to relatively low although not insignificant - levels. In contrast, South and South East Asia have high baseline mortality, and it remains high regardless of the future scenario. While sea-based strategies of adaptation reduce future mortality to around baseline levels, storm surge-associated mortality remains a major threat.

\section{Discussion}

\subsection{Mortality risk model}

We developed a new global-level storm surge mortality risk model. Methodologically, we made a number advances on the previous global-level work (McMichael et al., 2004, Dasgupta et al., 2009) which may provide the basis for further improvements (Table 2).

We were unable to validate the model due to a lack of data. The fit of the log mortality risk model suggested projections may be reliable, but mortality projections are clearly unreliable. One reason for this is that the model was fitted in the log space (see Online Resource, ESM, 
Appendix S7). Additionally, the particularities of any given country may introduce significant errors when estimating national-level average mortality using a global-level model. For instance, during the baseline period Bangladesh was struck by three events with exceptionally high mortality (CRED, 2011) meaning modelled average mortality was likely to be too low (which was the case; see Online Resource, ESM, table S7.1). Conversely, partly in response to these events, significant actions have been taken to reduce risk (Cash et al, 2013) potentially leading to overestimated average mortality. The former effect is due partly to chance and the latter is partly a feedback response. Future modelling efforts should attempt to address these influences.

This raises a more general issue related to the nature of surge deaths themselves: a few infrequent large events that are relatively unpredictable over decadal time scales cause the vast majority of mortality (CRED, 2011). This means average annual mortality is subject to discontinuities when a high mortality event occurs. In contrast, risk is a function of social and economic change as well as potential exposure; thus it tends to change continuously. While alternative discontinuous statistical approaches could be used to model mortality (e.g. Schoenberg, 2003)), we suggest modeling risk is preferable. That is, the ultimate purpose of modeling the health impacts of climate change is to avert them. To do this, risk - which is an evolving condition of daily life - must be reduced and models that trace its trajectory will best guide adaptation.

\subsection{Future mortality risk and mortality under climate change}

Our results are broadly consistent with previous assessments (McMichael et al., 2004). Climate change is expected to increase surge mortality, with the impacts concentrated in regions such as South and South-East Asia. Given the lack of model validation and the unreliability of the mortality estimates, our projections are best interpreted in terms of factors that appear to be important for impacts estimates, as this may provide guidance for future modelling.

For mortality risk, Bangladesh and Mozambique show how 'development' may initially increase risk. This behaviour was built into the model, but if it reflects reality, it reinforces the importance of implementing disaster risk reduction strategies now (see also Patt et al (2010)). In the USA, change in risk is driven by improved coping capacity (operationalized 
using population). However, this operationalization may be questionable, particularly in low income settings: conceivably, increased population may decrease coping capacity. The relation between population and risk, and how it varies with context, should be further investigated. Additionally, in equation (1) there is potentially overlap between the assumed influence of population on economies of scale in disaster management infrastructure (see 3.1) and land-based adaptation represented by the HDI. The current model treats these effects separately, but future work should investigate their interaction.

For mortality, the results suggest regions where climate change may significantly increase future mortality in the absence of sea-based adaptation. Further, they suggest that in some regions nationally-funded coastal defences could (if put in place) reduce mortality to relatively low (but still important) levels, while other areas may require external assistance to adapt. Given the long lead time required to put sea-based defences in place (around 30 years (Nicholls et al., 2007)), action needs to be taken in the near future. We suggest future health impact modelling should aim to assess the relation between mortality risk reduction and adaptation, and identify areas where mortality may remain intolerably high despite adaptation.

In sum, our results highlight the importance of considering climate change health impacts in the context of social, economic and demographic factors, all of which could both increase and decrease vulnerability to climate-related exposures.

\subsection{Limitations of the mortality risk model}

The major limitation of the model is the unreliability of the mortality estimates. This is partly due to the model being fit in the log space, 'outlier' countries, and the nature of surge mortality. An additional limitation is that the model was (necessarily) fit cross-sectionally but used to make estimates over time. This follows Patt et al's (2010) method and has precedent in previous climate-health modelling (e.g. McMichael et al, 2004). The underlying assumption is that statistical relations are causal, or at least stable over time. It is however plausible, for example, that the relation between land-based adaptation and the HDI will change with time. 
Two further limitations arise from assumptions regarding the baseline mortality data. Firstly, by standardizing mortality to national population in the year 2000 we account for population changes, but implicitly assume that change within a country is spatially uniform. As urban and coastal areas are growing more rapidly than rural areas, standardization may result in underestimates of mortality. While this assumption is consistent with DIVA, future work should attempt to account for spatial differentials.

Secondly, a time-series of mortality data was used to generate average annual mortality for a baseline time-slice, but this was regressed against the HDI specific to the year 2000 in the mortality risk model. This implicitly assumes current land-based strategies have been in place over the time period covered by mortality dataset and may underestimate their benefits. However, this effect is partially off-set because the long time-series of data included only 'big' events: observations suggest that socioeconomic improvements have a smaller benefit for high intensity compared to low intensity events (UNISDR, 2011).

Additional limitations are associated with factors not included in the model. For climate change, the model only considers sea-level rise. Future work should also consider changes in cyclone characteristics, particularly as intense events cause the majority of health impacts. This would involve closer integration of coastal flood and health models, data for exposure by event intensity, and development of quantitative knowledge of the lethality of surges of different intensities.

For health impacts, we only considered mortality. Coastal floods, however, also impact on morbidity, including injuries, infections, and mental health (Ahern et al., 2005). These impacts may be direct or indirect (e.g. via crop loss or damage to infrastructure), and immediate or delayed. Such complexities make the full recording and attribution of impacts difficult, and quantitative knowledge on which to base models is lacking. It may be possible to develop general quantitative relations linking surge, vulnerability, and morbidity risk for various outcomes, building on the limited attempts to date (Li et al., 2007, Fewtrell and Kay, 2008). 


\section{Conclusions}

Climate change is expected to worsen storm surge events and, in interaction with population vulnerability, this may have significant health impacts. While our model does not provide reliable mortality estimates we have made methodological innovations and recommendations for future model development. Further, we have illustrated the importance of socioeconomic factors in conditioning risk. In general, climate change health impacts work has tended to model physical aspects robustly but - partly due to data limitations - to model social and economic factors with considerably less rigour. To develop a stronger knowledge base for averting the health impacts of storm surge, as well climate change in general, conceptual and methodological innovations that robustly capture both physical and social factors are essential. 


\section{Tables and figures}

Table 1: Estimates of mortality risk equation parameters, $95 \%$ confidence intervals, $p$-values, and standardized regression coefficients.

\begin{tabular}{|c|c|c|c|c|c|}
\hline Variable & Parameter & $\begin{array}{c}\text { Mean } \\
\text { estimate }\end{array}$ & 95\% Confidence Interval & p value & $\begin{array}{c}\text { Standardized } \\
\text { regression } \\
\text { coefficients }\end{array}$ \\
\hline$E_{i}$ & $\beta_{1}$ & 1.73 & 0.75 to 2.72 & 0.001 & 0.25 \\
\hline$P_{i, j}$ & $\beta_{2}$ & -0.78 & -0.96 to -0.60 & 0.0001 & -0.63 \\
\hline$H_{i, j}$ & $\beta_{3}$ & 18.01 & 7.68 to 28.35 & 0.001 & 1.25 \\
\hline$H_{i j}^{2}$ & $\beta_{4}$ & -13.46 & -22.38 to -4.54 & 0.003 & -1.08 \\
\hline & $k$ & 15.60 & 11.51 to 19.69 & 0.0001 & \\
\hline
\end{tabular}

${ }^{1}$ The standardized regression coefficients quantify the change in the LHS of the equation relative to its standard deviation when a given RHS variable is changed by one standard deviation; the greater the absolute value of the statistic, the more responsive the LHS is to the variable. For example, 0.25 means that a 1 standard deviation change in $E_{i}$ is associated with a 0.25 standard deviation change in log mortality risk. 
Table 2: Methodological advances made in the mortality risk model and suggestions for further development

\begin{tabular}{|c|c|}
\hline Aspect & Advances and suggested ways forward \\
\hline $\begin{array}{l}\text { Baseline } \\
\text { mortality data: } \\
\text { time-period } \\
\text { covered }\end{array}$ & $\begin{array}{l}\text { Previous work was based on models fit using mortality data selected on a criterion of } \\
\text { 'completeness'. These data cover a relatively short time period over which infrequent, high } \\
\text { intensity events are effectively random. Yet these events cause the majority of mortality. } \\
\text { We have addressed this using an ad hoc method. Formal methods, perhaps Bayesian, should } \\
\text { be developed to allow the utilization of longer time-series. (For example, Reis and Stedinger } \\
\text { (2005) use such a method to generate flood frequency curves; this could be used as a basis } \\
\text { for work in health modelling). Additionally, when using long series of mortality data, the } \\
\text { associated population data should be standardized, as they were in this paper. }\end{array}$ \\
\hline $\begin{array}{l}\text { Baseline } \\
\text { mortality data: } \\
\text { surge-specific } \\
\text { deaths }\end{array}$ & $\begin{array}{l}\text { Previous work was based on models fit using mortality data selected on a geographic- (i.e. } \\
\text { 'coastal') rather than event-based (i.e. 'storm surge') definition. Further, all deaths } \\
\text { associated with a cyclone rather than only those attributable to storm surge were included. } \\
\text { To assess the potential mortality impacts of future sea-level rise, we separated surge-specific } \\
\text { deaths from other deaths in a given event using an ad hoc method. Further research is } \\
\text { needed on the relation between vulnerability and various causes of deaths in events, and/or, } \\
\text { consideration should be given to methods of including cause of death in event data. }\end{array}$ \\
\hline $\begin{array}{l}\text { Socioeconomic } \\
\text { change: } \\
\text { representation }\end{array}$ & $\begin{array}{l}\text { The distribution of almost all health outcomes is associated with socioeconomic conditions. } \\
\text { Previous work represented socioeconomic change using GDP/capita. However, this may be } \\
\text { a poor proxy for actual living conditions of the vulnerable population. } \\
\text { In this paper, we used the HDI, which is a better proxy and can be derived from existing } \\
\text { scenario data. Variables such as life expectancy and fertility are indicators of national living } \\
\text { conditions and are implicit in already available population projections. Further, fertility is } \\
\text { influenced by education levels; this data could also be made available. In the future, a } \\
\text { broader range of health-relevant socioeconomic projections are required. }\end{array}$ \\
\hline $\begin{array}{l}\text { Socioeconomic } \\
\text { change: } \\
\text { distribution of } \\
\text { benefits }\end{array}$ & $\begin{array}{l}\text { Previous work assumed that as GDP increases, socioeconomic conditions improve for all } \\
\text { people. However, benefits may accrue to certain groups, and evidence suggests vulnerability } \\
\text { to disasters may initially worsen with 'development' for some populations. } \\
\text { We have attempted to model vulnerability in terms of both the benefits and harms of } \\
\text { 'development' by using a quadratic relation. As the negative aspects of development may be } \\
\text { experienced by the most vulnerable groups in society, we suggest theoretically-grounded } \\
\text { methods for improved quantitative modelling should be developed. }\end{array}$ \\
\hline
\end{tabular}


Figure 1: The process of generating inputs for calibrating the mortality risk model, starting from data sources (shaded boxes ${ }^{1}$ ), via raw data (ovals) and its transformations (unshaded rectangular boxes), and finally to the variables used as inputs (striped boxes) into the mortality risk equation (double bordered box). See text for details .

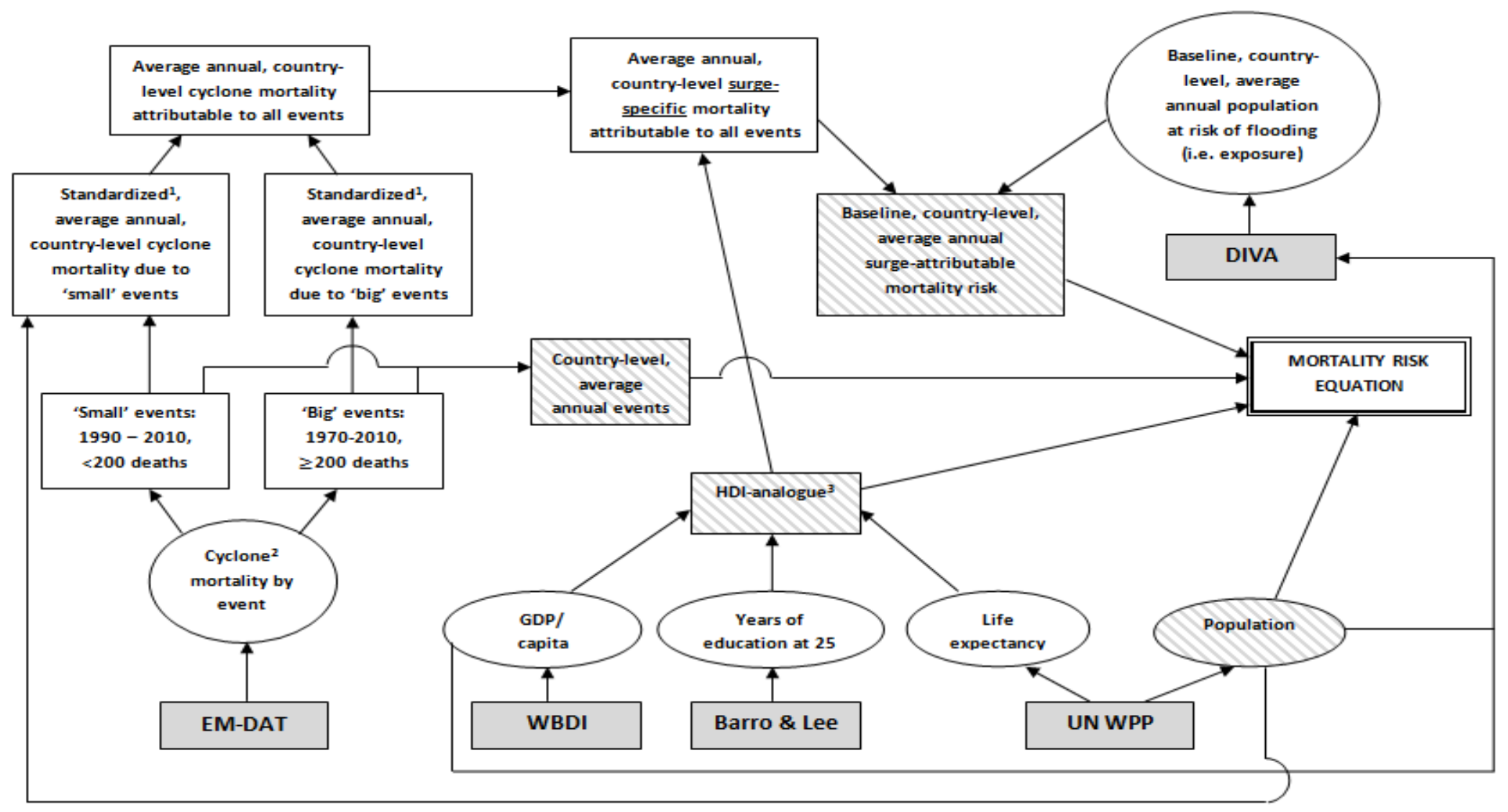

1 'EM-DAT' is Emergency Events Database; 'WDBI' is World Bank Development Indicators; 'Barro \& Lee' is Barro and Lee (2000); 'UNWPP' in United Nations World Population Prospects; 'DIVA' is Dynamic Interactive Vulnerability Assessment (coastal flood model).

2 'Standardized' refers to standardization to population in the year 2000; see text for details.

${ }^{3}$ We use 'cyclones' to refer to any cyclone-like event that may be associated with a storm surge (e.g tropical cyclones, extra-tropical storms).

${ }^{4}$ The HDI-analogue is a modified version of the Human Development Index; see text for details. 
Figure 2: Projected log mortality risk estimates made using equation 1, for four selected countries, as a function of the HDI-analogue ( $H_{i, j}$, x-axis) and national population ( $P_{i, j}, \mathrm{y}$-axis). The colour contours represent $\log$ mortality risk per million (LHS of equation 1), with blues corresponding to the lowest risk and reds to the highest. The black dots indicate log mortality risk for a given time-slice (shown in brackets). Each dot is labelled with projected average annual surge exposure ( $X_{i, j}$, un-bracketed numbers), which is a function of sea-level rise, land subsidence, and population living in the coastal zone. Results are for futures with climate change (A1b emissions) and land-based adaptation, but without sea-based adaptation (e.g. improved sea dikes).
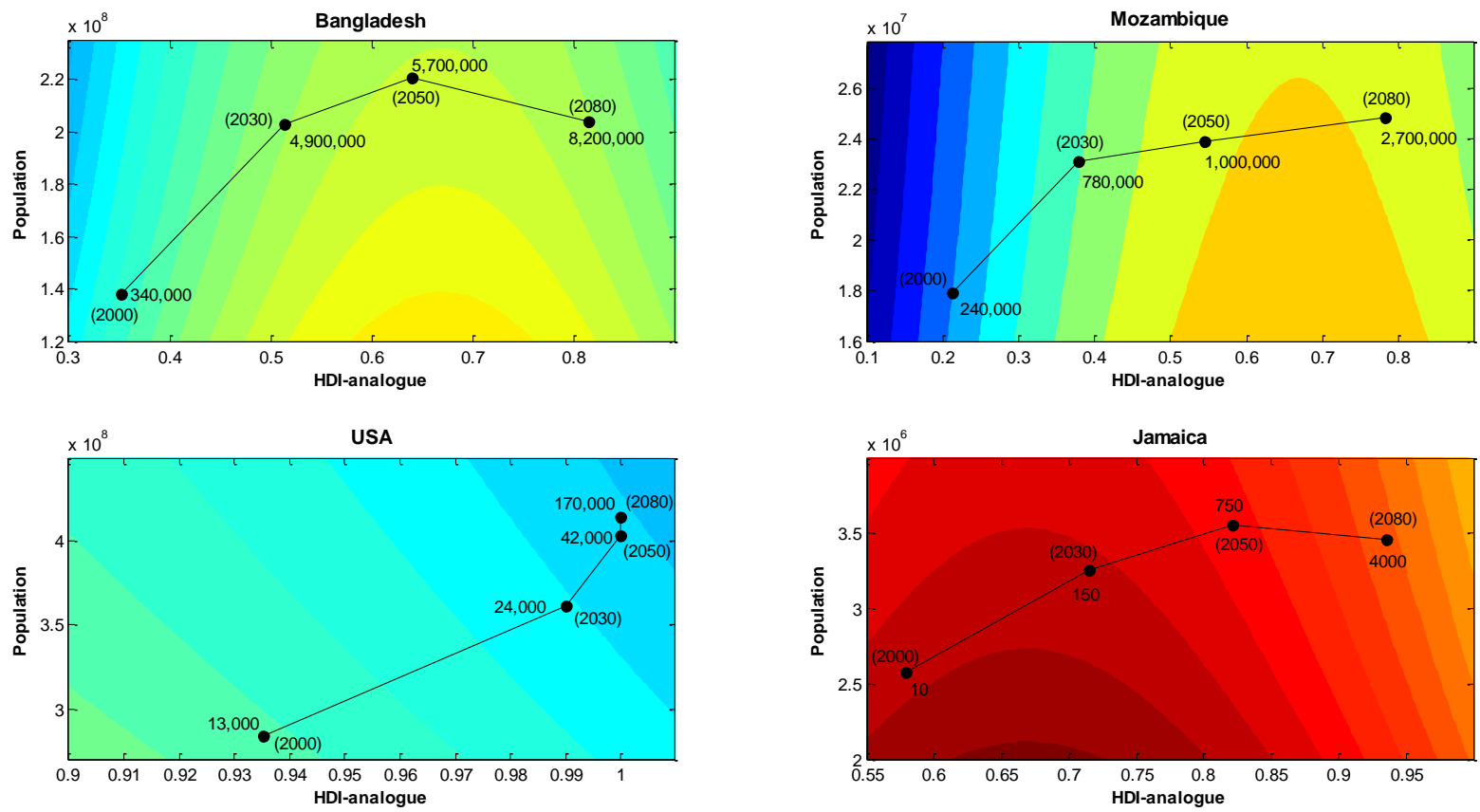
Figure 3: Estimates of regional-level ${ }^{1}$ average annual mortality ranges at baseline and in the 2030s, 2050s and 2080s (running left to right in the figure, as per bottom axis) based on median exposure estimates ${ }^{2}$. For each region, there are three coloured horizontal bars which, from top to bottom, are (i) a future without climate change or adaptation $^{3}$, (ii) a future with climate change but no adaptation, (iii) a future with climate change and adaptation. The colour of the bar indicates the range of average annual mortality as indicated in the legend on the right.

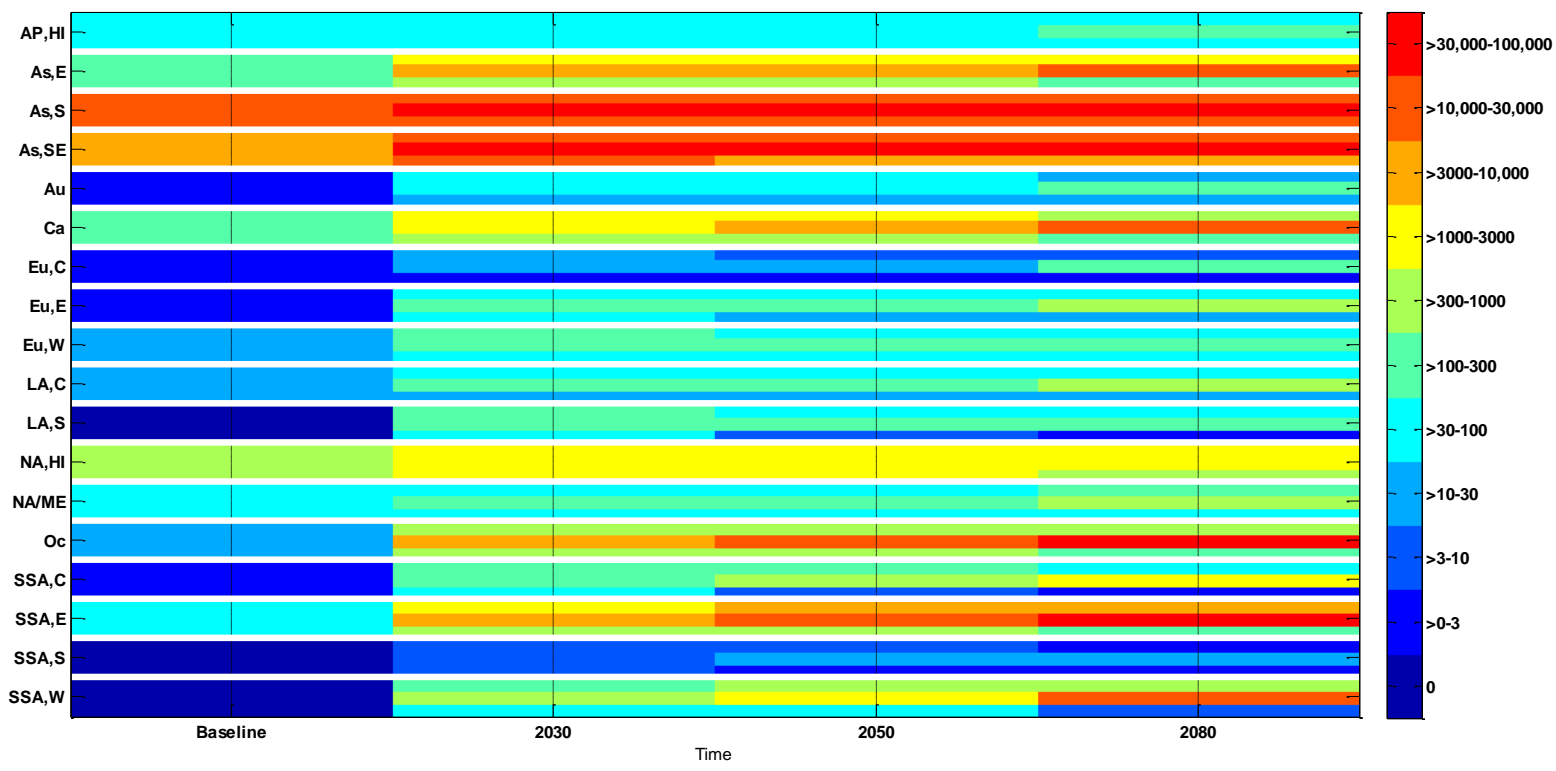

${ }^{1}$ Regions are As,HI: Asia Pacific, High Income; As,E: Asia, East; As,S: Asia, South; As,SE: Asia, Southeast; Au: Australasia; Ca: Caribbean; Eu,C: Europe, Central; Eu,E: Europe, Eastern; Eu,W: Europe Western; LA,C: Latin America, Central; LA,S: Latin America, South; NA,HI: North America, High Income; NA/ME: North Africa/Middle East; Oc: Oceania; SSA,C: Sub-Saharan Africa; SSA,E: Sub-Saharan Africa, East; SSA,S: Sub-Saharan Africa, Southern; SSA,W: Sub-Saharan Africa, West.

${ }^{2}$ Median exposure is the median estimate based on 7 GCMs; see text for details.

${ }^{3}$ Adaptation specifically refers to improved sea-defences; in all scenarios land-based defences improve as the Human Development Index increases (see text for further details). 